\title{
Impact of a Half Dome Cable Permitting Process on Search and Rescue Activity, Hiker Mortality Rates, and Operational Costs Above Little Yosemite Valley
}

\author{
Susanne J Spano, MD ${ }^{1}$; John A Seymer, BS ${ }^{2}$; Desiree H Crane, DO ${ }^{1}$; Paul S Auerbach, MD, MS ${ }^{3}$ \\ ${ }^{1}$ University of California, San Francisco (Fresno), Fresno, CA; ${ }^{2}$ National Park Service, Hawaii National Park, HI; ${ }^{3}$ Stanford University School of \\ Medicine, Stanford, $C A$
}

Introduction-The summit of Yosemite's Half Dome is reached using cable handrails for the final $146 \mathrm{~m}$ (480 ft). Access to these cables was restricted to users with permits in 2010. The authors aim to describe the impact of permitting on search and rescue (SAR) in the region of the park most affected by permitting.

Methods-An observational study from 2005 to 2009 and 2011 to 2015 comparing the number of incidents, major incidents (exceeding \$500), victims, and fatalities before and after permitting the use of cable handrails on Half Dome in the area above Little Yosemite Valley (LYV) and parkwide. Each year was analyzed separately with $t$ tests and Mann-Whitney $U$ tests. Data are presented as mean \pm SD.

Result-The number of hikers in the study area was reduced by up to $66 \%$ by permitting. Above LYV from 2005 to 2009, there were 85 SAR incidents, 134 victims, 8 fatalities, 38 major incidents, and annual SAR costs of $\$ 44,582 \pm 28,972$. From 2011 to 2015 , the same area saw 54 SAR incidents, 156 victims, 4 fatalities, 35 major incidents, and annual SAR costs of $\$ 27,027 \pm 19,586$. No parameter showed statistical significance. Parkwide SAR incidents decreased from 232 to 198 annual incidents $(P=0.013)$ during the same time period, with parkwide mortality increasing from 8 to 12 deaths annually $(P=0.045)$.

Conclusions - SAR incidents, victims, fatalities, or costs above LYV did not decrease after cable handrail permitting. Parkwide SAR activity decreased during the same intervals. This strongly suggests that overcrowding is not the key factor influencing safety on Half Dome. This discordant trend warrants close observation over 5 to $10 \mathrm{y}$.

Keywords: preventative search and rescue (PSAR), wilderness trauma, injury prevention, overcrowding, medical response, wilderness first aid

\section{Introduction}

Half Dome is a monolithic granite dome rising $2682 \mathrm{~m}$ $(8800 \mathrm{ft})$ above sea level. It is reached via a 23 to $26 \mathrm{~km}$ (14-16 $\mathrm{mi})$ round-trip hike with an elevation gain of 1463 m (4800 ft). The Half Dome Trail splits midway into the John Muir Trail and Mist Trail, which converge

Presented as an oral research presentation at the Society for Academic Emergency Medicine (SAEM) Annual Conference, May 18, 2017, Orlando, FL.

Corresponding author: Susanne J Spano, MD, University of California, San Francisco (Fresno); 155 N Fresno Street, Suite 206, Fresno, CA 9370; e-mail: sspano@gmail.com.

Submitted for publication February 2018.

Accepted for publication November 2018. at Nevada Falls to create a single route through Little Yosemite Valley (LYV) to Half Dome (Figure 1). Twenty-five percent of individuals needing search and rescue (SAR) services in Yosemite National Park (YNP) are on trails leading towards Half Dome. ${ }^{1}$

In 2010, the Half Dome management plan addressed visitor safety concerns through the issuance of permits, mid-May through mid-October (conditions permitting),

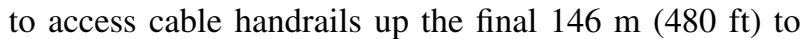
the summit of Half Dome. Technical climbers, who descend via the cable handrails, are not required to have a permit. The cable handrails are laid flat on the granite during the winter season to prevent destruction from snow slides (Figure 2); permits are not issued when the cables are down. 


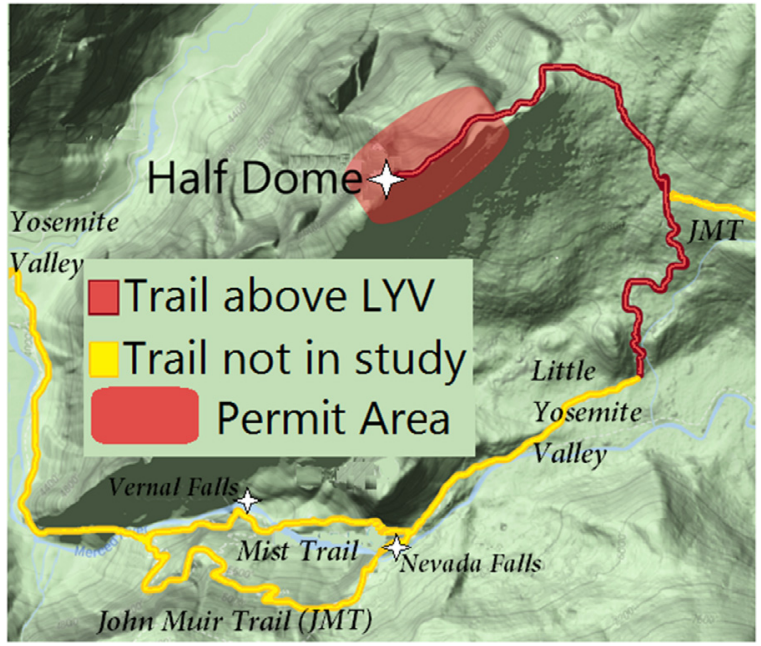

Figure 1. Half Dome Trail map. The Half Dome Trail splits midway into the John Muir Trail and Mist Trail, which converge at Nevada Falls to form a single route through LYV to Half Dome. Permit area is the restricted section including subdome, the cable route, and summit. Figure adapted from NPS file created by Jared Doke on August 3, 2010 and Paul Doherty on August 12, 2010 using NAD 1983 UTM Zone $11 \mathrm{~N}$.

Climbing aids are not unique to YNP. Angel's Landing in Zion National Park has a permanent chain-assisted section for the last $800 \mathrm{~m}(0.5 \mathrm{mi})$ of a $4 \mathrm{~km}(2.4 \mathrm{mi})$ hike that does not require a permit. The Zion National Park website documents 7 deaths on the Angel's Landing trail since the park's establishment in 1919, without indicating if these occurred at the chain-assisted section. Denali National Park has fixed snow pickets to Denali Pass and from camp 14 up to the West Buttress, requiring a mountaineering specialty permit and backcountry permit. From 1903 to 2006, there were 96 deaths on Denali, $45 \%$ of which were due to falls, with fatalities decreasing by $53 \%$ after climber registration was established in $1995 .^{2}$

Wilderness permits and trailhead quotas in US national parks provide opportunities for solitude, as required by the Wilderness Act. ${ }^{3}$ The authors know of no other similar type of intervention intended to protect visitors in the wilderness. Per the internal park communication authorizing emergency regulatory provisions to limit visitors, available online, the push for permitting came after 7 fatal incidents on Half Dome in the 2000s, up from 3 fatalities each decade in the 1980s and 1990s. ${ }^{4}$ A recent study conflicts with this accounting, with 8 documented deaths in the 1980s. ${ }^{5}$ Thirty-two fatalities have occurred on Half Dome over the last $85 \mathrm{y}$, including a fall from the cables in May 2018; however, less than $20 \%$ of these have been related to activity on the cable handrails. ${ }^{5}$
Hikers asked to analyze photographs of the cable route perceived an increased risk when the number of people at one time (PAOT) on the cable handrails exceeded 70 persons. ${ }^{6}$ Consulting reports have demonstrated significant decreases in PAOT on the cable handrails, subdome, and Half Dome summit after permitting. ${ }^{6,7}$ Although an intuitive proxy for individual vulnerability, PAOT is not a validated measure of adverse events. Using the PAOT measure, permitting has been described as effective. ${ }^{8,9}$

To the authors' knowledge, permitting as a method of preventative SAR has not been studied using outcomesbased data rather than a surrogate marker (PAOT). Our goal with this study was to compare SAR incidents, mortality, and costs before and after implementation of Half Dome cable permitting.

\section{Methods}

The authors chose for the study area Half Dome Trail, above LYV, $2.3 \mathrm{~km}(1.4 \mathrm{mi})$ past the convergence of trails above Nevada Falls (Figure 1). The LYV cutoff is a common location indicator on SAR reports (above LYV, below LYV). Hikers without a permit to summit Half Dome are unlikely to proceed beyond LYV, with the exception of long-distance backpackers on the $354 \mathrm{~km}$ John Muir Trail (220 miles) who chose not to add a Half Dome permit to their through-hike. Specifically excluded were the preceding John Muir Trail and Mist Trail because these popular trails were not likely to see a significant decrease in use.

The comparison periods were "before permits" of 2005 to 2009 and "after permits" of 2011 to 2015. The year of 2010 was excluded because permits were issued only on weekends and holidays. Impact was described as changes in pre- and post-permitting of SAR call volume, costs, major responses (exceeding $\$ 500$ per the National Park Service [NPS]), and morbidity and mortality from LYV to Half Dome summit. Nature, location, and complexity of the rescues were secondary outcomes. Hikers, climbers, and base jumpers were all included because each would interface with the cable handrails and have the potential to affect safety. Per capita data were not calculated because the PAOT reports available were underpowered to predict use over an 11-y study period. Yearlong data were obtained to compare trends in the study area to parkwide metrics over the same time periods. Yearlong data were also used to calculate any changes in off-season incident frequency.

All YNP SAR activities are electronically documented either through direct entry or digital uploads of paper reports into the Incident Management Analysis and Reporting System. Because of multiple transitions in cataloging, data were manually searched. In December 

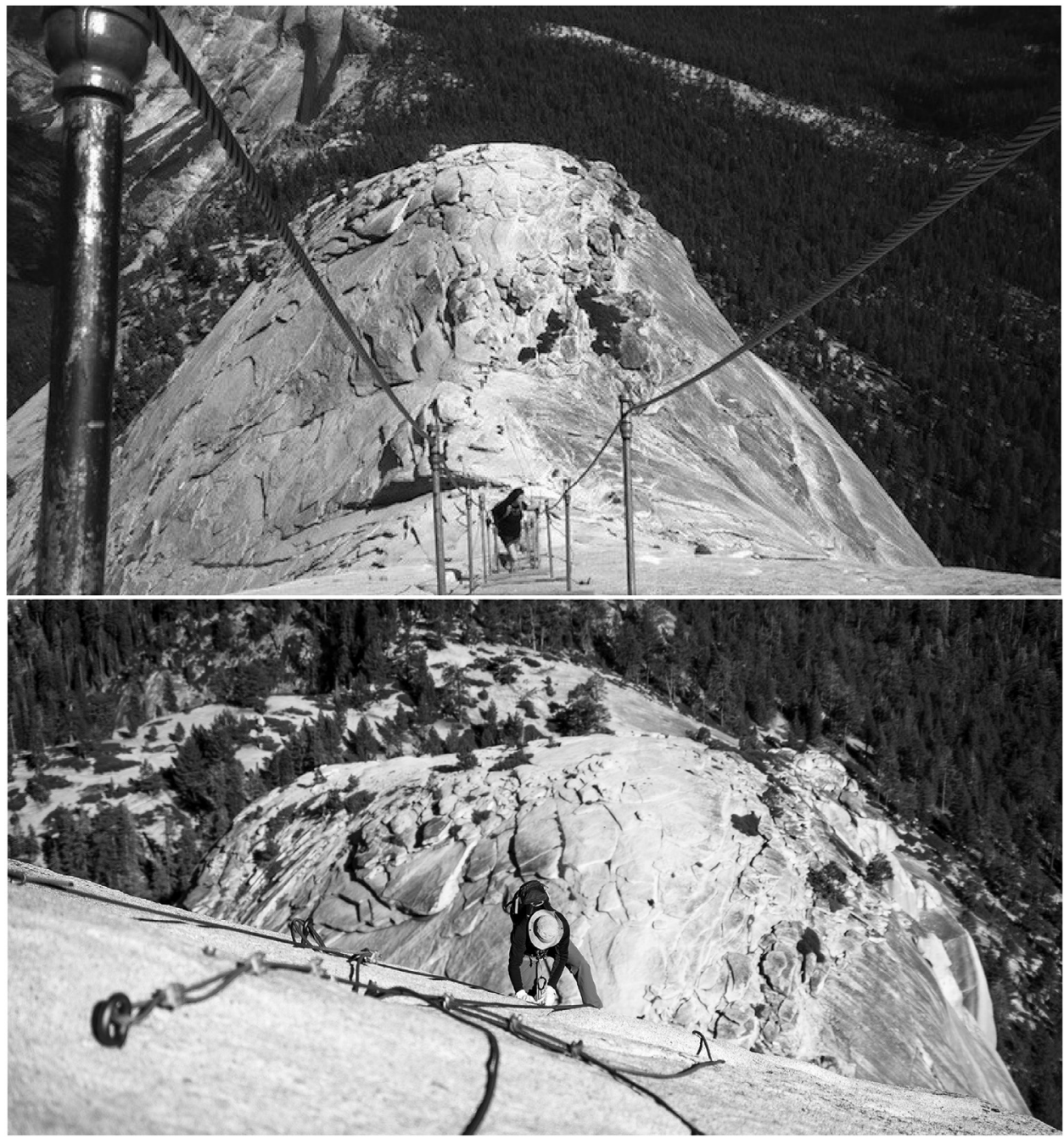

Figure 2. Cables in the upright position (May-September) and down position (off-season). When upright, the cables literally create handrails with wooden foot supports. In the off-season, the metal stanchions and wooden slats are removed to protect them from damage secondary to winter conditions. Photographs reproduced with permission from Kim Dinan/Brian Sutton and Outdoor Project/Basil Newburn, respectively.

2015, one author (JS), an NPS ranger, identified all reports on the Half Dome Trail over the past $11 \mathrm{y}$, out of approximately 2200 SARs. Each of the 750 relevant records was searched for an indication that the response occurred at or above LYV.

Data extracted from these reports included nonidentifying demographic information, date, weather, location, nature of emergency, cost, number of affected parties, method of evacuation, and mortality. Parkwide SAR data from year-end reports are stripped of patient identifiers; annual reports were collected for the study period 2005 to 2015.

Descriptive narratives pertaining to each incident's cause, rescue, and location were categorized by one author (SS). Fifty-four illness/injury labels were categorized into 4 broad categories: trauma, medical, behavioral, or environmental. Events were categorized by presumed root cause. For example, if an avalanche caused a fall, "environmental" rather than "trauma" would be the category; without the avalanche, it cannot be asserted that a traumatic incident would ensue. Ten subtypes of rescue modalities were recategorized in 5 categories referring to the highest-level intervention: ranger assist, bystander/self-rescue, horse-out, helicopter, or search. Eighteen location descriptors were consolidated into 3: climber, trail, and permit area. Climber refers to areas accessible only by technical climbing. Trail refers to any part of the Half Dome Trail above LYV and below the ranger permit checkpoint. Permit area is the restricted section, approximately $640 \mathrm{~m}$ (0.4 miles), including subdome, the cable route, and the summit (Figure 1). 
Each year of data was statistically managed as a separate case with $t$ tests and Mann-Whitney $U$ tests (as applicable) comparing pre- and post-permitting of SAR call volume, costs, major responses, mortality, and categorical descriptor frequencies using SPSS Statistics. Statistical significance was accepted at $P<0.05$. Data are presented as mean \pm standard deviation (range), as appropriate. The study received institutional review board approval from the University of California, San Francisco (Fresno) Medical Education Program.

\section{Results}

Above LYV, a total of 140 SAR incidents involving 290 persons and 12 fatalities occurred during the years 2005 to 2015, excluding the transition year of 2010. Every year of data above LYV is listed individually with a comparison to parkwide data (Table 1). The majority of incidents $(P<0.001)$ occurred when the cables were up in the summer season (Table 2). Before permitting, there were 85 SAR incidents, 134 victims, 8 fatalities, and 38 major incidents above LYV. Post-permitting, excluding 2010, there were 54 SAR incidents, 156 victims, 4 fatalities, and 35 major incidents above LYV. There were no statistical differences in injury patterns, rescue modalities, costs, or mortality before and after the permitting of cable access, so categories of rescue etiologies and evacuations are presented as a cohort for simplicity.

SAR victims above LYV were male (66\%), $31 \pm 13$ y old (8-70), and most frequently sought assistance for a trauma-related complaint ( $41 \%$ trauma, $31 \%$ medical, $19 \%$ behavioral, and $10 \%$ environmental), with rescues costing an average of $\$ 2530(\$ 0-\$ 23,819)$ per incident. There was no difference in the types of evacuation before or after permitting, with helicopter rescue $(33 \%)$ being the most frequently used. This percentage includes 2 evacuations of multiple stranded visitors on the summit: 41 witnesses to a fatal cable handrail fall during a hailstorm (2009, pre-permitting) and 78 persons instructed to evacuate during a wildfire (2014, post-permitting). SAR calls above LYV were most likely to occur on Friday,
Table 2. Summer season vs off-season SAR incidents above LYV before and after permits

\begin{tabular}{lccc}
\hline Year & Summer season & Off-season & P value \\
\hline 2015 & 7 & 0 & $<0.001$ \\
2014 & 5 & 1 & \\
2013 & 7 & 1 & \\
2012 & 19 & 0 & \\
2011 & 11 & 5 & \\
2010 & 22 & 2 & \\
2009 & 16 & 1 & \\
2008 & 22 & 1 & \\
2007 & 20 & 3 & \\
2006 & 12 & 1 & \\
2005 & 8 & 1 & \\
\hline
\end{tabular}

SAR, search and rescue; LYV, Little Yosemite Valley.

Saturday, or Sunday, both before and after permitting (Figure 3). Wet weather played a role in only 9 incidents. Weather data for the incident dates show no significant difference in the number of rainy days or windiness, but the average temperature during rescues in the post-permit years $(2011-2015)$ was warmer $(P<0.001)$ than in the pre-permit years. The distribution of SAR incidents across climber, trail, and permit area was significantly greater in the permit area $(P=0.038)$ after permitting. There were no significant differences in injury category before or after permitting. These relationships can be visualized in Table 3.

The subtypes of trauma, medical, behavioral, and environmental complaints can be seen in Table 4 . Trauma responses (67 incidents) included lower extremity injuries (75\%), falls from a height (19\%), and minor and/or unspecified trauma (4\%). Medical illnesses (51 incidents) were described as symptoms, not diagnoses: nausea/vomiting (35\%), syncope/near-syncope or loss of consciousness without return to baseline (25\%), unspecified medical complaint (18\%), cardiac-related (10\%), diabetes-related $(8 \%)$, anaphylaxis $(2 \%)$, and cardiac arrest (2\%). Behavioral problems (31 incidents) included

Table 1. Study area (vs parkwide) SAR activity by year from 2005-2015

\begin{tabular}{lcclllllllll}
\hline Year & 2015 & 2014 & 2013 & 2012 & 2011 & 2010 & 2009 & 2008 & 2007 & 2006 & 2005 \\
\hline SARs & $6(216)$ & $6(181)$ & $8(175)$ & $19(215)$ & $16(202)$ & $24(245)$ & $17(242)$ & $23(248)$ & $23(238)$ & $13(219)$ & $9(214)$ \\
Subjects & $8(276)$ & $84(287)$ & $8(203)$ & $21(248)$ & $35(226)$ & $29(278)$ & $60(283)$ & $25(269)$ & $26(270)$ & $14(252)$ & $9(238)$ \\
Fatalities & $1(15)$ & $0(11)$ & $0(9)$ & $0(9)$ & $3(15)$ & $1(7)$ & $2(9)$ & $1(5)$ & $3(9)$ & $1(9)$ & $1(9)$ \\
Major SARs & $5(116)$ & $4(86)$ & $2(81)$ & $13(95)$ & $11(88)$ & $15(114)$ & $8(115)$ & $9(98)$ & $9(100)$ & $8(97)$ & $4(87)$ \\
Cost & $25(464)$ & $13(322)$ & $4(365)$ & $41(556)$ & $52(539)$ & $59(694)$ & $69(535)$ & $23(504)$ & $80(842)$ & $39(357)$ & $12(1159)$ \\
\hline
\end{tabular}

SAR, search and rescue.

Major SARs are defined as SAR incidents with operational costs exceeding $\$ 500$. All costs are noted in thousands of US dollars. 


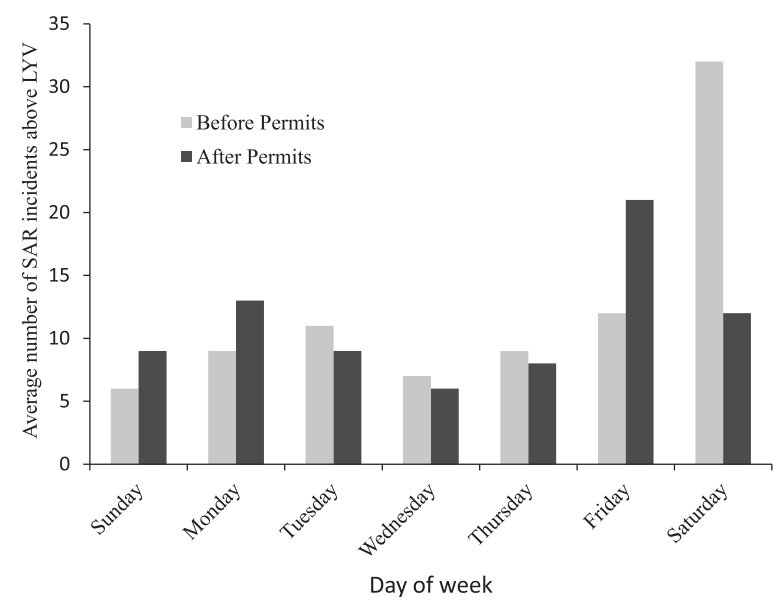

Figure 3. Number of average SAR incidents above LYV by day of week before and after permitting access to the cable handrails leading to the Half Dome summit. SAR calls above LYV were most likely to occur on Friday, Saturday, and Sunday. After permitting, the incident spike on Saturdays was more evenly distributed.

lost/separated parties (61\%); hikers afraid of falling from the cables, summit ledge, or on wet rock (16\%); suicide (13\%); illegal base jumping (6\%); and 1 false report (3\%). Environmental issues (16 incidents) were attributed to heat/dehydration (63\%), followed by single cases (6\%) of avalanche, altitude illness, rattlesnake bite, hypothermia, wildfire hazard, and lightning fatality resulting in a fall from the cable handrails.

None of the financial indicators reached statistical significance. Before permitting in the area above LYV from 2005 to 2009 , the average annual SAR cost was $\$ 44,582$ with an average cost per incident of $\$ 2786$. After permitting from 2011 to 2015, the same area above LYV saw an average annual SAR cost of $\$ 27,027$ with a decrease in average incident cost to $\$ 2502$. Parkwide average SAR volume decreased from 232 incidents per year before 2010 to 198 incidents annually after 2010 $(P=0.013)$. Despite this significant drop in overall SAR volume, parkwide mortality increased from 8 to 12 deaths annually, on average, after $2010(P=0.045)$.

\section{Discussion}

The background article for permitting access to Half Dome cites the need to address weekend crowding to prevent accidents and simultaneously implicates wet weather as the cause of nearly all cable-related accidents. ${ }^{4}$ After a recent fatal fall from the cable handrails in May 2018, wet weather was again implicated as the causative agent in most falls. ${ }^{10}$ The data from our study do not support either of these logical assertions. Wet weather played a role in only 9 incidents, without a change in frequency before or after permitting. No significant decrease in absolute numbers of SAR activity above LYV has been evidenced post-permitting. Using the maximum of 117 PAOT on the Half Dome cablehandrails from a 2010 non-permit day and a maximum of 51 PAOT on a 2010 permit day, ${ }^{7}$ it can be extrapolated that the $56 \%$ reduction in individuals present should scale down SAR incidents and victims (and costs) by at least $50 \%$. These numbers conservatively underestimate the reduction of visitors using published data. The baseline mean daily use of 692 users daily on weekends and holidays from 2008 (pre-permits) estimates a maximum of 146 PAOT on the cable route. ${ }^{7}$ The busiest day of 2009 saw 1300 persons attempt a summit. ${ }^{8}$ A regression analysis on observed PAOT supports the

Table 3. Demographics of SAR incidents above LYV before and after permits

\begin{tabular}{|c|c|c|c|}
\hline Variable & Before permits (2005-2009) & After permits (2010-2015) & $\mathrm{P}$ value \\
\hline Temperature (high) & $22^{\circ} \mathrm{C}\left(72^{\circ} \mathrm{F}\right)$ & $25^{\circ} \mathrm{C}\left(78^{\circ} \mathrm{F}\right)$ & 0.000 \\
\hline Rainy incidents & 4 & 5 & 0.778 \\
\hline Permit area & $18(21 \%)$ & $20(36 \%)$ & 0.038 \\
\hline Trail area above LYV & $63(74 \%)$ & $30(54 \%)$ & \\
\hline Climber area & $4(5 \%)$ & $6(11 \%)$ & \\
\hline Trauma & $40(47 \%)$ & $21(38 \%)$ & 0.562 \\
\hline Medical illness & $24(28 \%)$ & $18(32 \%)$ & \\
\hline Behavioral issue & $14(17 \%)$ & $9(16 \%)$ & \\
\hline Environmental harm & $7(8 \%)$ & $8(14 \%)$ & \\
\hline Helicopter rescue & $27(32 \%)$ & $18(32 \%)$ & 0.356 \\
\hline Bystander/self-rescue & $19(22 \%)$ & $16(29 \%)$ & \\
\hline Horse-out rescue & $19(22 \%)$ & $9(16 \%)$ & \\
\hline Ranger assist rescue & $13(15 \%)$ & $12(21 \%)$ & \\
\hline Search team rescue & $7(8 \%)$ & $1(2 \%)$ & \\
\hline
\end{tabular}

SAR, search and rescue; LYV, Little Yosemite Valley. Bolding denotes significance. 
Table 4. Types of injury and illness above LYV

\begin{tabular}{llll}
\hline Trauma $(n=67)$ & Medical $(n=51)$ & Behavioral $(n=31)$ & Environmental $(n=16)$ \\
\hline Leg $(75 \%)$ & Nausea/Vomiting (35\%) & Lost $(61 \%)$ & Heat/Dehydration $(63 \%)$ \\
Falls $(19 \%)$ & Altered state $(25 \%)$ & Fear of falling $(16 \%)$ & Avalanche $(6 \%)$ \\
Unspecified (4\%) & Unspecified $(18 \%)$ & Suicide $(13 \%)$ & Rattlesnake bite $(6 \%)$ \\
& Cardiac $(10 \%)$ & Base jumping $(6 \%)$ & Altitude illness $(6 \%)$ \\
& Diabetes (8\%) & False report (3\%) & Hypothermia (6\%) \\
& Anaphylaxis $(2 \%)$ & & Wildfire hazard $(6 \%)$ \\
& CPR (2\%) & & Lightning $(6 \%)$ \\
\hline
\end{tabular}

LYV, Little Yosemite Valley; CPR, cardiopulmonary resuscitation.

post-permit cable PAOT estimates of 40 to 60 when 400 permits are issued. ${ }^{11}$

If anything, the use of permits appears to have increased the individual risk for a SAR incident in the permit area. The same raw number of incidents and victims spread over $56 \%$ fewer participants would indicate a doubling of relative risk. We do not have an explanation for this observation. It is possible that the exclusivity of obtaining a permit increases motivation or risk-taking behavior for the individual attempting to summit, generating more SAR activity if physical state or other indicators of personal hazard were ignored because this was their "one chance." The publicity generated by the permit system might attract a broader audience with a different fitness or experience profile. The Sierra Club states more than 30,000 applicants applied for Half Dome permits in 2014 , with only a $20 \%$ individual likelihood of success. Since the conclusion of this study period, YNP reduced by 100 the number of available daily permits. The rationale for this additional reduction was not provided.

Our study found medical illness (32\%), lower extremity injuries (31\%), lost person (10\%), and vomiting (9\%) as the top causes for rescue calls above LYV, with no difference in reason for rescue calls before or after permitting. An impact on cable-related incidents was not observed. Among parkwide incidents from 1990 to 1999 , the most common causes for SAR services were lower extremity injuries (34\%), lost person (15\%), and dehydration or hunger $(8 \%){ }^{1}$ The next decade, top causes were extremity injuries (53\%), gastrointestinal problems $(11 \%)$, and altered mental status $(10 \%)$; the search rate was not provided. $^{12}$ Our study confirms extremity injuries and medical/gastrointestinal illnesses as persistent issues over 3 decades of tracking rescue activity in YNP.

Containing costs for uncompensated SAR activity is important. The cost of a SAR incident is influenced by the Incident Commander's allocation of internal and external resources. Major incidents, 38 pre-permitting vs 35 postpermitting, cost more than $\$ 500$ and are paid for by the federal NPS budget instead of the individual park budget. YNP has previously ranked first of all NPS units in SAR costs, consuming $25 \%$ of the federal NPS budget as it accounted for $10 \%$ of all national SAR events. ${ }^{13}$ Our study demonstrated $\$ 2600$ per incident above LYV, a sharp drop from $\$ 4400$ in the 1990 s. ${ }^{1}$ Underreporting bias should have made it more likely to demonstrate a significant difference post-permit. Helicopter evacuation of 78 people from the summit of Half Dome post-permitting was largely funded through the state fire budget and was not reflected in the yearly SAR cost, which is yet another contributor to difficulty interpreting these data. Absence of a statistically significant cost savings in this study underscores the need to normalize reporting to make valid comparisons across time.

\section{LIMITATIONS}

There potentially exist individual factors influencing SAR outcomes, such as risky behavior or unquantified physical exhaustion, that cannot be controlled for and are unlikely to be affected by the presence or absence of crowding. Catastrophic events may also be stochastic occurrences. This limitation would not affect the accuracy of our data, but if these factors are the principle etiology for SAR responses above LYV, they would not be accounted for by our experimental design.

Etiology and status of medical conditions were recorded by rangers who may have had limited diagnostic abilities, particularly in an austere environment. Constitutional physical symptoms can be ambiguous, affecting the calculated proportions of cardiac or altitude-related illness. The use of broad categorizations (trauma, medical, environmental, and behavioral) was meant to address the limitations inherent to the nuanced and variable presentations of medical conditions. 
Granular detail of the results may have been lost as a result of data simplification by using categorical classifications or transitions in recordkeeping during the study period. The authors attempted to show broad trends rather than analyze individual incident detail, which lessens the concerns these issues present.

Uncertainty regarding the true number of participants prevents a calculation of an incidence rate of SAR activity before and after permitting. This may limit the external validity of the findings in YNP to other areas where permitting might be considered. The variance between the average use estimates, of 700 persons on weekends and 400 on weekdays ${ }^{14}$ and the maximum documented 1300 users $^{8}$ on a single day, makes any pre-permit per capita calculations unreliable. Although it is known that 400 permits were issued during the latter half of the study, this does not take into account nonuse, cancellations, illegal use, and descending climbers. A future prospective study using an automated visitor counter at the base of subdome or the cables could make reliable per capita calculations possible.

These limitations do not undermine our finding of absence of an anticipated decrease in SAR incidents, costs, or mortality despite reducing the number of users of Half Dome.

\section{Conclusions}

Implementation of permitting access to Half Dome cable handrails reduced the number of people able to summit an iconic landmark, but the data do not support a significant reduction in the overall toll of associated human suffering, mortality, or SAR costs above LYV. This strongly suggests that overcrowding is not the key factor influencing safety on Half Dome. PAOT thresholds identified by hikers have not been validated as a reliable surrogate marker for safety in this setting. Lower extremity injuries and medical illnesses, notably gastrointestinal upset, have been prevalent problems leading to SARs for visitors to YNP, and the area above LYV in our study was consistent. Based on these data, the focus on crowding on the cable handrails as the main driver of SAR activity may not be accurate. The exclusivity of a Half Dome cables permit (20\% success rate) may reframe its significance to the possessor as a license to proceed, regardless of circumstances that may have a greater influence on visitor safety than adverse weather or crowding. Prospective studies are warranted to evaluate whether limiting use of the cable handrails leads to detrimental effects for the permit holders and to better identify the variables having the greatest impact on mortality and cost. One consideration for managers of natural resources reviewing this work is to complement current wilderness permitting systems with rescue card registration. This would help financially support local search and rescue efforts, while also giving an opportunity for education on common rescues that have occurred in that area.

Acknowledgments: The authors thank Lisa Hendy for help in obtaining access to Yosemite Search and Rescue data, Claudine Ronay for assisting in compiling financial data, Svetlana Bagdasarov for assisting as liaison with the institutional review board, and Deanna M. Halliday for statistical analysis.

Author Contributions: Study concept and design (SS); data acquisition (JS); data analysis (SS, JS); manuscript draft (SS, JS, DC, PA); manuscript critical revision (SS, JS, DC, PA); manuscript final approval (SS, JS, DC, PA).

Financial/Material Support: None.

Disclosures: None.

\section{References}

1. Hung E, Townes D. Search and rescue in Yosemite National Park: a ten year review. Wilderness Environ Med. 2007;18(2):111-6.

2. McIntosh SE, Campbell AD, Dow J, Grissom CK. Mountaineering fatalities on Denali. High Alt Med Biol. 2008;9 (1):89-95.

3. US Department of Justice. The Wilderness Act of 1964. Available at: https://www.justice.gov/enrd/wilderness-act1964. Accessed August 24, 2018.

4. National Park Service. Half Dome background paper. Yosemite, CA. 2010. Available at: https://www.nps.gov/ yose/planyourvisit/upload/halfdomemanagement.pdf. Accessed October 15, 2018.

5. Richardson G, Spano S. Death on the dome: epidemiology of recreational deaths on Half Dome in Yosemite National Park. Wilderness Environ Med. 2018;29(3):338-42.

6. Lawson S, Choi J, Reigner N, Newman P, Gibson A. Half Dome Cables Modeling and Visitor Use Estimation Final Report: Yosemite National Park. White River Junction, VT: Resource Systems Group; 2009.

7. Lawson S, Kiser B, Reigner N. Half Dome Cables Visitor Use Model Scenario Analysis Final Report. White River Junction, VT: Resource Systems Group; 2011.

8. Lawson S, Newman P, Choi J, Pettebone D, Meldrum B. Integrated transportation and user capacity research in Yosemite National Park. Transp Res Rec. 2009;2119 (2119):83-91.

9. Reigner N, Lawson S, Meldrum B, Pettebone D, Newman P, Gibson A, et al. Adaptive management of visitor use on Half Dome, an example of effectiveness. J Park Recreat Admi. 2012;30(3):64-78.

10. Warszawski M. How to be safe on the Half Dome cables. Hiking Half Dome is always a challenge. Making a hard choice could save your life. The Fresno Bee. May 242018. Available at: https://www.fresnobee.com/opinion/opn-columns-blogs/marek-warszawski/article211865119.html. Accessed September 6, 2018. 
11. Pettebone D, Meldrum B, Leslie C, Lawson SR, Newman $\mathrm{P}$, Reignerd N, et al. A visitor use monitoring approach on the Half Dome cables to reduce crowding and inform park planning decisions in Yosemite National Park. Landsc Urban Plan. 2013;118:1-9.

12. Boore S, Bock D. Ten years of search and rescue in Yosemite National Park: examining the past for future prevention. Wilderness Environ Med. 2013;24 (1):2-7.
13. Heggie T, Amundson M. Dead men walking: search and rescue in U.S. national parks. Wilderness Environ Med. 2009;20(3):244-9.

14. Woita R, Leslie C, Meldrum B. Half Dome trail visitor use monitoring report. Resources Management and Science Division, Visitor Use and Social Sciences Branch: Yosemite National Park. 2012. Available at: https://www.nps. gov/yose/learn/nature/upload/hdmonitoring2012.pdf. Accessed May 31, 2018. 